\title{
Evaluation of the effectiveness and efficiency of the triage emergency department nursing protocol for the management of pain
}

This article was published in the following Dove Press journal: Journal of Pain Research

16 October 2017

Number of times this article has been viewed

\author{
Loris Butti' \\ Olga Bierti' \\ Raffaela Lanfrit' \\ Romina Bertolini' \\ Sara Chittaro' \\ Stefania Delli Compagni' \\ Davide Del Russo' \\ Rossella Letizia Mancusi ${ }^{2}$ \\ Franco Pertoldi' \\ 'S.O.C. Emergency Medicine, AAS3 \\ Ospedale Sant'Antonio di San Daniele \\ del Friuli, ${ }^{2}$ C.R.E.A. Sanità, University \\ of Tor Vergata, Rome, Italy
}

Correspondence: Rossella Letizia Mancusi C.R.E.A. Sanità, University of Tor Vergata, Piazza A. Mancini, 4, 00196 Rome, Italy

Tel +393289041084

Email rosella.letizia.mancusi@uniroma2.it
Introduction: Pain is a common symptom presented in the emergency department (ED) although it is often underestimated, poorly evaluated and treated. The application of a protocol for timely pain management ensured by the nurse can avoid the delays in the analgesic treatment and improve the patient's quality of waiting.

Aims: To check the effectiveness and efficiency of the protocol aimed at early pain management in triage, active in our ED. In particular, the response to analgesic treatment was evaluated 60 minutes after the administration and at discharge. Patient satisfaction was also evaluated using two anonymous questionnaires both at discharge and 48 hours later via telephone.

Methods: A single-center, observational study was conducted on a prospective cohort of patients (aged $\geq 4$ years) with a pain symptom at admission in ED with no surgical picture.

Results: In the observation period (June 2015-May 2016), 382 patients were enrolled, and of these, $312(84.8 \%)$ accepted pain therapy during triage stage in the ED. In $97.4 \%$ of the cases, orosoluble paracetamol $1000 \mathrm{mg}$ was administered. In the re-evaluation done 60 minutes later, $65.9 \%$ of the patients showed a reduction of at least 2 points on Numeric Rating Scale (NRS), equal to a mean reduction of 2.24 points (95\% CI: $2.03-2.45)$. The mean time of analgesia intake was equal to 5.9 minutes (95\% CI: 3.8-8.1). In the re-evaluation done at discharge, $33.2 \%$ of the patients showed a reduction of NRS score $>50 \%$, leading to a mean reduction of $39 \%$ (95\% CI: $35.3 \%-41.9 \%)$. The level of patient satisfaction was high with a mean value $>9$ points (maximum satisfaction $=10$ ).

Conclusion: This protocol shows that optimal pain management was achieved by patients rapidly receiving an effective painkiller therapy at triage, leading to substantial patient satisfaction. In moderate pain, orosoluble paracetamol $1000 \mathrm{mg}$ provided a reduction of NRS score by 2 points in $67.6 \%$ of the patients, confirming to be the analgesic of choice in ED.

Keywords: pain management, protocol, triage, analgesic, orosoluble paracetamol

\section{Introduction}

A correct and timely pain assessment and management are crucial aspects in the area of emergency services. Indeed, numerous studies have shown that there was a pain symptomatology, as a primary or accompanying symptom, in an extremely high proportion of patients who were admited to an emergency department (ED) (from $50 \%$ to $79 \%)^{1}$ as well as the repeated detection of an inadequate and untimely oligoanalgesia.,3 The literature reports that in $\sim 40 \%$ of the patients who accessed the ED, pain was not treated properly and efficiently due to numerous critical factors such as logistical, organizational as well as cultural. ${ }^{4,5} \mathrm{~A}$ review of the literature 
related to emergency pain management ${ }^{6}$ identified multiple causes for its poor management, in particular the absolute lack of recognition of the pain, the lack of tools to assess the intensity of pain at admission as well as the lack of tools to assess the adequacy of the treatment administered (monitoring of pain during the ED stay), and the failure to adopt and share specific guidelines. In addition, there are cultural barriers to both health care professionals and patients: opiophobia, the fear that the analgesia masks the signs and symptoms for diagnosis, and nonrecognition of the pain as a real emergency.

Furthermore, it should be considered that the constant overcrowding of the ED often leads to long waiting times, especially if it is a code with low caring priority, be a contributing cause to a lack of timeliness in pain assessment and treatment. ${ }^{7}$

This occurs despite the continuous development and provision of tools, guidelines and educational and training strategies. ${ }^{8,9}$

In our ED (Ospedale Sant'Antonio di San Daniele del Friuli-UD), in recent years, early management of pain has been a key challenge from both organizational and cultural perspective. In particular, a training program was carried out, aiming at increasing the knowledge about pain management among nurses and doctors, as well as a revision and implementation of a specific protocol for its management in triage. The study aimed to assess its validity in terms of both effectiveness and efficiency.

\section{Ethics statement}

The study was approved by the Ethics Committee (Comitato Etico Regionale Unico del Friuli Venezia Giulia) and was conducted according to the principles of the Helsinki Declaration. Patients enrolled in the study signed an informed consent form, and for patients who were not of legal age informed consent was signed by legally authorized representative.

\section{Study design and patient population}

A prospective, noninterventional, single-center study. The study enrolled the patients aged above 4 years with emergency-urgency admission to ED with painful symptoms that do not suggest the surgical intervention, in which case alternative routes were being planned. Table 1 provides the inclusion and exclusion criteria.

The main objective of the study was to verify, in normal clinical practice, the response in terms of reduction of the painful symptomatology in the patients treated according to the protocol for the nursing management of the pain during

Table I Inclusion/exclusion criteria

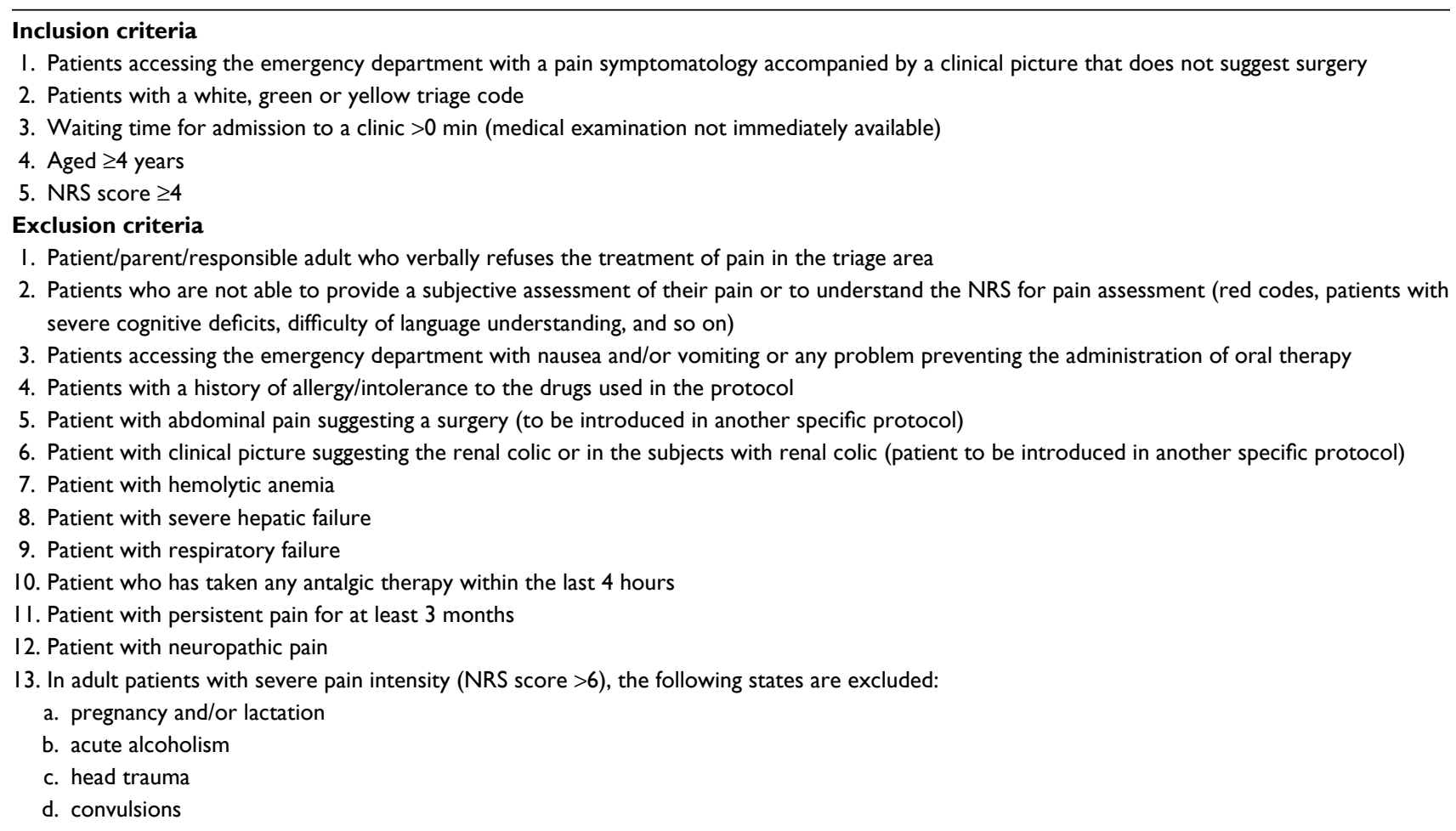

14. Patient on therapy with Furazolidone-Furoxone (treatment of infective diarrhea)

Abbreviation: NRS, Numeric Rating Scale. 
triage (Table 2). The following secondary endpoints were also evaluated: time of intake of the analgesia and degree of patient satisfaction.

The data were collected using a dedicated paper form, completed by the nurse of triage during the triage phase and administration of the therapy, and by the doctor/nurse during the stay in ED until the discharge.

For the evaluation of the pain intensity, Numeric Rating Scale (NRS) was used for the patients aged over 8 years. The NRS ranges from a minimum score of 0 to 10 , where the extremes represent the absence of pain and the maximum pain experience, respectively. For the subjects under the age of 8 years, the algometric evaluation and the Wong-Baker scale were used.

At the time of discharge from the $\mathrm{ED}$, all eligible patients were asked to express their level of satisfaction with regard to the management of painful symptomatology. The instrument used is divided into seven items (courtesy and willingness to listen, competence and professionalism demonstrated, attention paid to the needs and problems, clarity of the information received, completeness of the information received, mode of administration of the drug, and respect for privacy). A subsequent verification of the overall level of satisfaction in terms of the assistance and therapy received was carried out 48 hours after the discharge via telephone interviews. The instrument used consists of five items (overall satisfaction on the effectiveness of the treatment and care received, easy access to care and treatment, waiting times, drug distribution by the structure at the time of discharge, and satisfaction of the analgesic therapy received/distributed at the time of discharge). For both the instruments and each individual item, the degree of satisfaction was measured on a numerical scale from 0 to $10(0=$ minimum satisfaction and $10=$ maximum satisfaction (Table $\mathrm{S} 1$ ).

\section{Statistical analysis}

All statistical analyses were conducted considering the eligible patients. For the sociodemographic parameters at the time of enrolment, the clinical variables related to the admission to the ED including the time of receipt of analgesia, a descriptive analysis was carried out: mean, standard deviation, range of variation, median, interquartile interval and number of missing data, 95\% CI (for continuous variables); absolute frequency, percentage, $95 \%$ $\mathrm{CI}$ and the number of missing data for discrete variables. While the correlations across qualitative variables were measured using the chi-square test, the differences across quantitative variables were evaluated using Student's $t$-test. For statistical analysis, the SPSS ${ }^{\circledR}$ Statistics Version 20.0.0 package was used.

\section{Results \\ Baseline characteristics}

In the observation period from June 3, 2015, to May 23, 2016, a total of 382 patients were enrolled and 14 of these patients were excluded from the analysis because they did not meet the inclusion criteria, having a mild painful symptomatology (NRS score $<4$ ). Therefore, there are 368 eligible patients, of which $312(84.8 \%)$ accepted the intake of therapy in the triage area in therapy vs $56(15.2 \%)$ who refused it. Table 3 reports the enrolment characteristics of 312 patients who adhered to the protocol.

Overall, a higher proportion of women (57.1\%) with a mean age obviously older was observed; 52.8 women vs 45.3 men ( $t$-test resulted significant; $P=0.001)$. Overall, 171 patients $(54.8 \%)$ access the ED for traumatic or contusionrelated pain and 81 of these patients were men, equal to $60.4 \%$ of all men who have adhered to the therapy and 90

Table 2 Nursing management protocol for patients with pain

\begin{tabular}{|c|c|c|}
\hline Pain intensity & Adults (> 12 years) & Children \\
\hline $\begin{array}{l}\text { Moderate } \\
4-6 \text { NRS }\end{array}$ & $\begin{array}{l}\text { - Orodispersible paracetamol } 1000 \mathrm{mg} \\
\text { (>12 years old with body weight }>40 \mathrm{~kg})\end{array}$ & $\begin{array}{l}\text { - Orodispersible paracetamol } 250 \text { mg } \\
\text { (Between } 4 \text { and } 8 \text { years old, weighing between } 17 \text { and } 25 \mathrm{~kg} \text { ) }\end{array}$ \\
\hline $\begin{array}{l}\text { Severe } \\
7-10 \text { NRS }\end{array}$ & - Morphine I vial $5 \mathrm{~mL} / 10 \mathrm{mg}$ & $\begin{array}{l}\text { - Orodispersible paracetamol } 500 \mathrm{mg} \\
\text { (Between } 9 \text { and } 12 \text { years old, weighing between } 26 \text { and } 40 \mathrm{~kg} \text { ) } \\
\text { - Orodispersible paracetamol } 1000 \mathrm{mg} \\
\text { (>12 years old with body weight }>40 \mathrm{~kg} \text { ) }\end{array}$ \\
\hline To exclude & $\begin{array}{l}\text { Hypersensitivity, pregnancy, lactation, acute alcoholism, } \\
\text { head trauma, convulsions, respiratory failure, severe } \\
\text { hepatic failure, Furazolidone-Furoxone therapy for the } \\
\text { treatment of infectious diarrhea }\end{array}$ & $\begin{array}{l}\text { Hypersensitivity, hemolytic anemia, severe hepatic failure, } \\
\text { respiratory failure }\end{array}$ \\
\hline
\end{tabular}

Abbreviation: NRS, Numeric Rating Scale. 
Table 3 Enrolment characteristics of the patients who adhered to the protocol (eligible patients with the administration of antalgic therapy in the triage area)

\begin{tabular}{|c|c|}
\hline Enrolment characteristics & n (\%) \\
\hline Sex & 312 \\
\hline Male & $134(42.9)$ \\
\hline \multicolumn{2}{|l|}{ Age (years) } \\
\hline Mean (SD) & $49.6(19.2)$ \\
\hline Median & 49.3 \\
\hline Min-Max & $14-92$ \\
\hline \multicolumn{2}{|l|}{ Weight (kg) } \\
\hline Mean (SD) & $75(15.5)$ \\
\hline Median & 74 \\
\hline Min-Max & $45-132$ \\
\hline \multicolumn{2}{|l|}{ Reason for admission } \\
\hline Trauma/contusion & $17 \mid(54.8)$ \\
\hline Musculo-articular pain & $60(19.2)$ \\
\hline Other pain & $35(11.2)$ \\
\hline Lumbar pain & $32(10.3)$ \\
\hline Otalgy & $5(1.6)$ \\
\hline Odontalgy & $4(1.3)$ \\
\hline Throat pain & $2(0.6)$ \\
\hline Headache/migraine & $2(0.6)$ \\
\hline Menstrual pain & $\mathrm{I}(0.3)$ \\
\hline \multicolumn{2}{|l|}{ NRS score } \\
\hline NRS 4 & $4(1.3)$ \\
\hline NRS 5 & $27(8.7)$ \\
\hline NRS 6 & $40(12.8)$ \\
\hline NRS 7 & $76(24.4)$ \\
\hline NRS 8 & $73(23.4)$ \\
\hline NRS 9 & $33(10.6)$ \\
\hline NRS 10 & $59(18.9)$ \\
\hline Moderate pain (NRS score 4-6) & $72(23.1)$ \\
\hline Severe pain (NRS score $>6$ ) & $240(76.9)$ \\
\hline
\end{tabular}

Abbreviations: NRS, Numeric Rating Scale; Max, maximum; Min, minimum. were women, equal to $50.6 \%$ of all female patients. Lumbar and musculoarticular pain was more pronounced in the women (33.1\% of women vs $24.6 \%$ of men). There are very few number of cases concerning the other planned clinical cases, for this reason, we proceeded to a recoding of the variable. It has been left off the pain from trauma or contusion (54.8\%), while we have grouped both lumbar pain and muscle-articular pain (29.5\%) in a single mode and finally under the diction, other pains (15.7\%) are included all other planned clinical pictures. Figure 1 shows the distribution of the clinical presentation at admission grouped according to the above criteria for the age of patients (in classes). It is shown that the traumatic/contusion-related pain is present in all age groups with values ranging from $12.3 \%$ (reported for the age groups 26-35 years and 56-65 years) to 24\% (for the age group $>65$ years), whereas for lumbar and musculoskeletal pains, this increases with increasing age up to the maximum that can be reached in patients over 65 years with $34.8 \%$, the $\chi^{2}$ test being significant $(P=0.006)$.

There is no statistically significant gender imbalances with respect to the distribution of pain intensity at admission, considering the score grouped as moderate pain (NRS score from 4 to 6) and severe pain (NRS score $>6$ ).

When each single NRS score for moderate pain $(4,5,6)$ and severe pain $(7,8,9,10)$ is separately analyzed, the results show an overlapping distribution between the two genders for the moderate pain, whereas a statistically significant difference is observed in severe pain $(P=0.003)$. In severe pain,

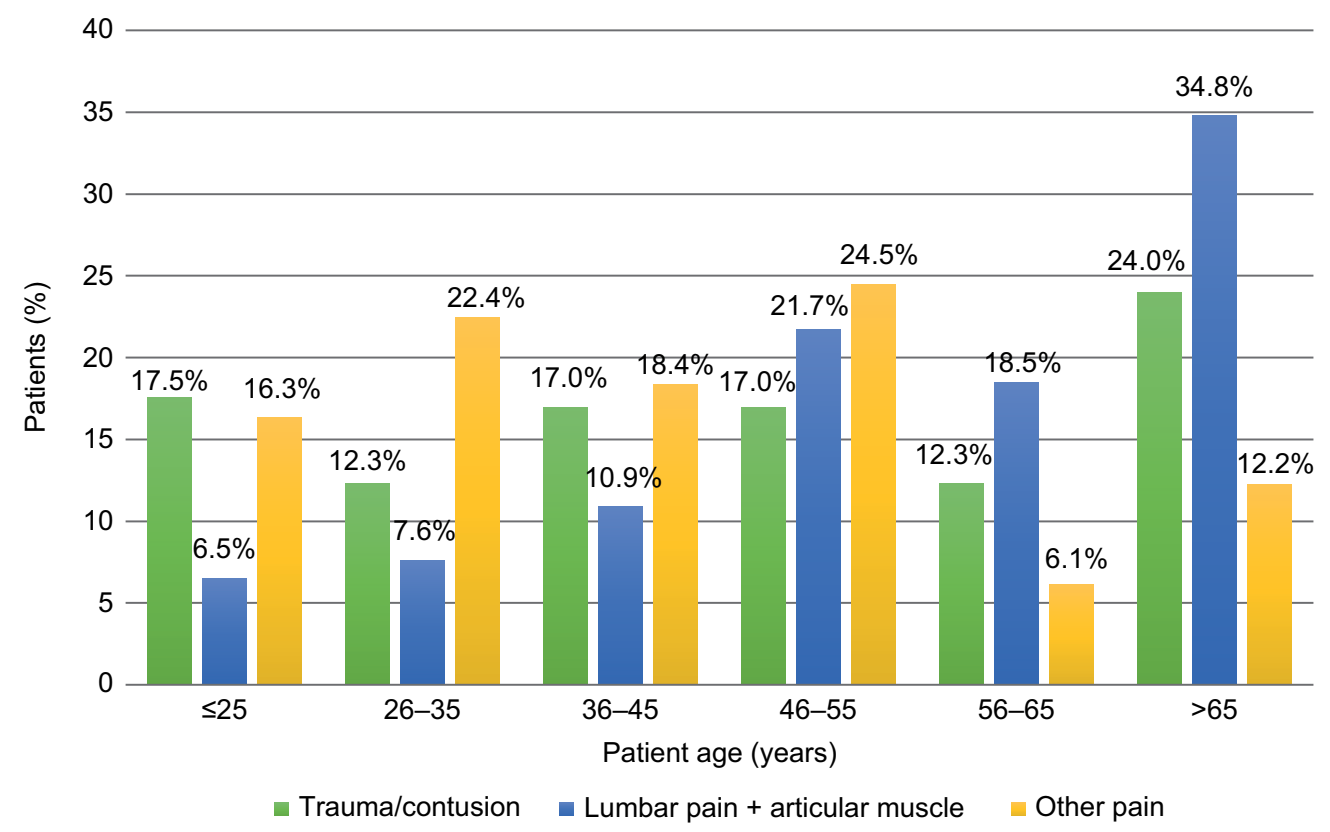

Figure I Reason for admission (grouped) by age classes. 
there is an imbalance of the male gender toward the lower limit of the interval (NRS score $=7$ ) and of the female gender toward the higher NRS scores of severe pain. In particular, for the maximum value, a difference of $7 \%$ is observed $(21.9 \%$ men vs $14.9 \%$ women with NRS = 10). The intensity of pain does not vary by age.

Table 4 reports the distribution of frequency according to the diagnosis made by the doctor at discharge. In particular, there are no particular deviations compared to the diagnosis at admission.

No significant differences were found between the genders even if a proportionally higher number of female cases were observed for nontraumatic pain, lumbar pain and arthrosis/arthritis-related pain; by contrast, in the pain resulting from trauma/contusion, case reports are more numerous among the men. Concerning the distribution by age, we can see that fractures are prevalently concentrated among patients aged over 65 years.

\section{Effectiveness verification}

Three hundred twelve patients who accepted to receive analgesic therapy in the triage area were administered orosoluble paracetamol $1000 \mathrm{mg}$ (orosoluble Tachipirina $1000 \mathrm{mg}$ ) in $97.1 \%$ of the cases, only eight patients $(2.6 \%)$ were treated with morphine (Oramorph $10 \mathrm{mg} / 5 \mathrm{~mL}$ ) and one subject was administered orosoluble paracetamol $250 \mathrm{mg}$ (orosoluble Tachipirina $250 \mathrm{mg}$ ).

When only the pain assessment at discharge is reported in the paper form, this was also considered as the main objective, with a tolerance not greater and lower than 10 minutes (evaluation at min. 50 minutes and at max. 70 minutes after the intake of analgesic therapy). A total of 201 patients (65.9\%) showed a reduction in the pain symptomatology of at least 2 points on the NRS, whereas for 99 patients $(32.5 \%)$ there was a reduction of $<2$ points and for only five patients $(1.6 \%)$ a worsening of pain was observed. For seven patients, the pain was not reevaluated at 60 minutes after the administration. The reduction of the

Table 4 Diagnosis at discharge

\begin{tabular}{llll}
\hline & Frequency & Percentage & Valid percentage \\
\hline Trauma/contusion & 114 & 36.5 & 37.7 \\
Nontraumatic pain & 96 & 30.8 & 31.8 \\
Fracture & 39 & 12.5 & 12.9 \\
Lumbar pain & 31 & 9.9 & 10.3 \\
Arthrosis/arthritis & 12 & 3.8 & 4.0 \\
Gonalgia & 10 & 3.2 & 3.3 \\
Valid cases & 302 & 96.8 & 100.0 \\
Missing & 10 & 3.2 & \\
Total & 312 & 100.0 & \\
\hline
\end{tabular}

painful symptomatology, as defined in the primary objective, does not appear to be conditioned by the intensity of pain at admission; for both classes (moderate and severe pain), the proportion of the patients who show a reduction of at least 2 points on the NRS is, however, higher than $65 \%(67.6 \%$ in the subjects with moderate pain at admission and $65.4 \%$ in the subjects with severe pain at admission, Figure 2).

The response to treatment, that is a reduction of at least 2 points on the NRS, does not vary by gender, age and the type of pain registered at admission (Figure 3), although there is a greater response in pain resulting from trauma or contusion where as many as $67.3 \%$ of the cases showed a reduction of at least 2 points on the NRS 60 minutes after the analgesic treatment.

Figure 4 shows the distribution by entity of pain reduction on NRS following the diagnosis made at the time of discharge. Though not statistically significant, a more pronounced achievement of the objective is observed in the cases of fractures $(71.1 \%)$ and nontraumatic pain, where about $70 \%$ shows a reduction in pain intensity by at least 2 points of NRS. The achievement of the objective is lower in the lumbar pain in osteoarthritis/arthritis and gonalgia, all types of pain likely attributable, in large part, to chronic/ persistent pain and/or pain with inflammatory component.

Table 5 shows the analysis on the distributions of NRS scores determined at the admission, at 60 minutes after taking the analgesic drug and at discharge, carried out using the Student's $t$-test for paired samples.

Comparisons are highly significant, and especially the difference between the NRS score at admission and the NRS score at 60 minutes after intake shows a mean difference of 2.24 points between the scores (95\% CI: 2.03-2.45; $P<0.0001)$. This indicates that an early treatment of pain according to the provisions of the study protocol guarantees, with a reliability of $95 \%$, a mean reduction of pain by at least 2 points on the NRS. The same comparison made vs the detection at discharge indicates a mean reduction of pain by at least 3 points (95\% CI: $2.74-3.29 ; P<0.0001)$.

\section{Efficiency verification}

A total of 308 cases are evaluable; the mean time to the intake of analgesia is 5.9 minutes, whereas the median time is equal to 2 minutes, and the maximum time to intake is equal to 189 minutes (3 hours and 9 minutes).

Table 6 shows the mean times to intake of analgesic therapy by reason for admission. A greater variability occurs in the diagnosis at discharge (Table S2), even if the comparison between the median values, however, shows that $50 \%$ 


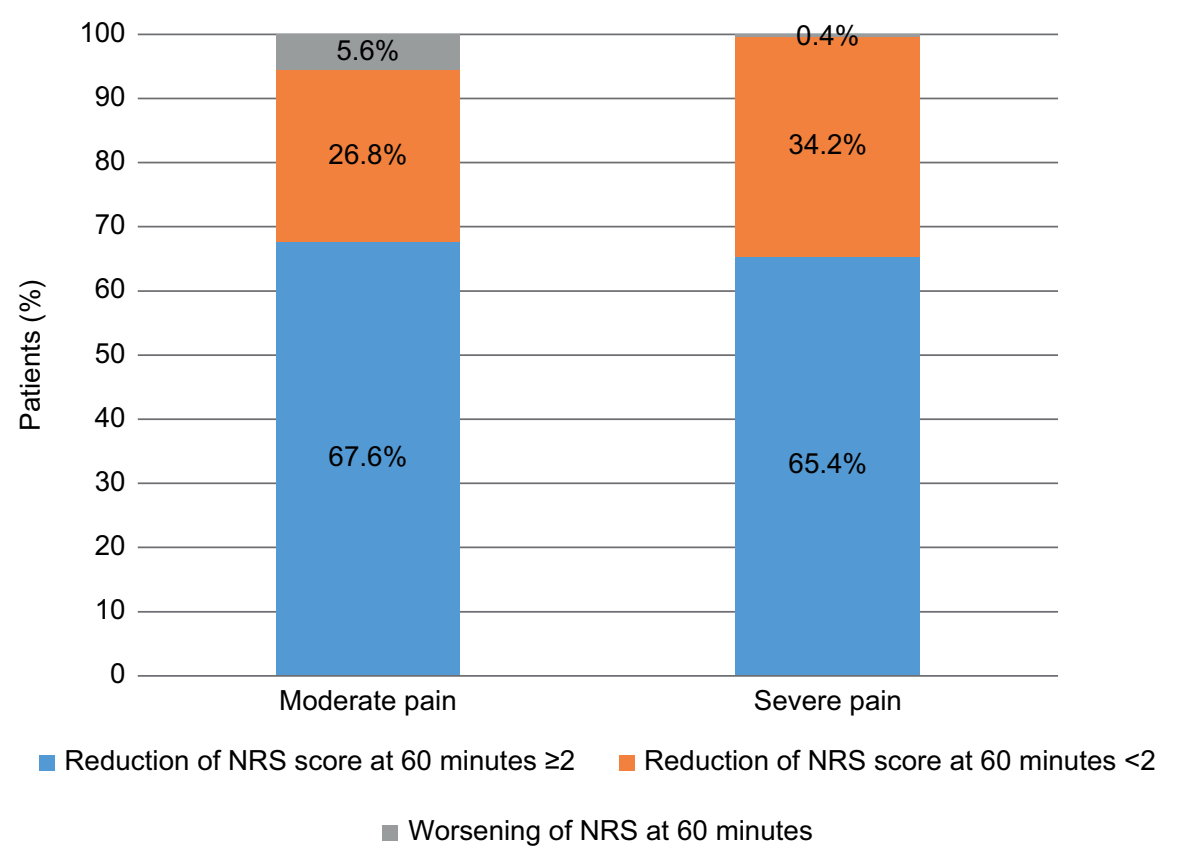

Figure 2 Reduction of the pain according to the intensity of the pain NRS indicated at admission. Abbreviation: NRS, Numeric Rating Scale.

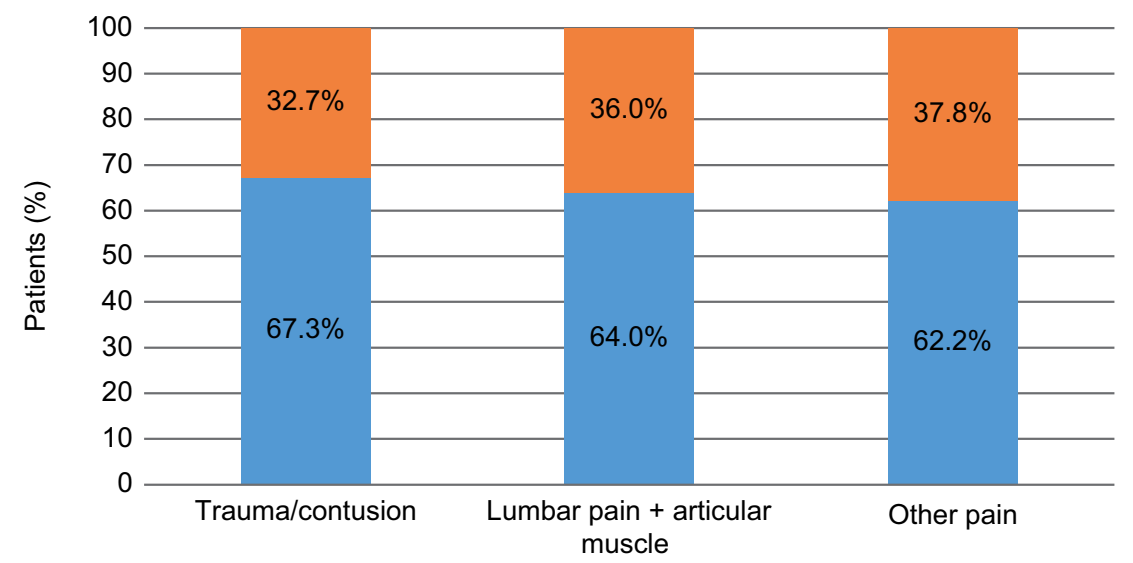

- Reduction of NRS score at 60 minutes $\geq 2$

Reduction of NRS score at 60 minutes $<2$

Figure 3 Reason for admission (grouped) by the reduction of pain on NRS Abbreviation: NRS, Numeric Rating Scale.

of patients are treated within 2 minutes after the admission, irrespective of diagnosis.

Concerning the patient satisfaction level at the time of discharge (Figure 5), all the items under examination examined show a score higher than 9 (maximum 10), meaning that $50 \%$ of the patients expressed the highest degree of satisfaction for all the items. Given the sum of the satisfaction ratings (maximum 70), the mean value is equal to 65.26 , whereas the median is equal to 69 points, indicating a high level of patient satisfaction at discharge.

Concerning the satisfaction level detected at 48 hours after the discharge via telephone interview (Figure 6), considering the sum of the satisfaction level ratings at 48 (maximum 50) hours after the discharge, the mean value is equal to 44.16 while the median value corresponds to 45 points, indicating a high level of patient satisfaction even at 48 hours after the discharge.

\section{Discussion}

A first consideration concerning the verification of effectiveness and efficiency of the protocol used in the study is the high patient compliance. In fact, as many as $84.2 \%$ of the eligible patients were willing to receive the antalgic therapy during the triage, a datum clearly greater than that found in the literature, 


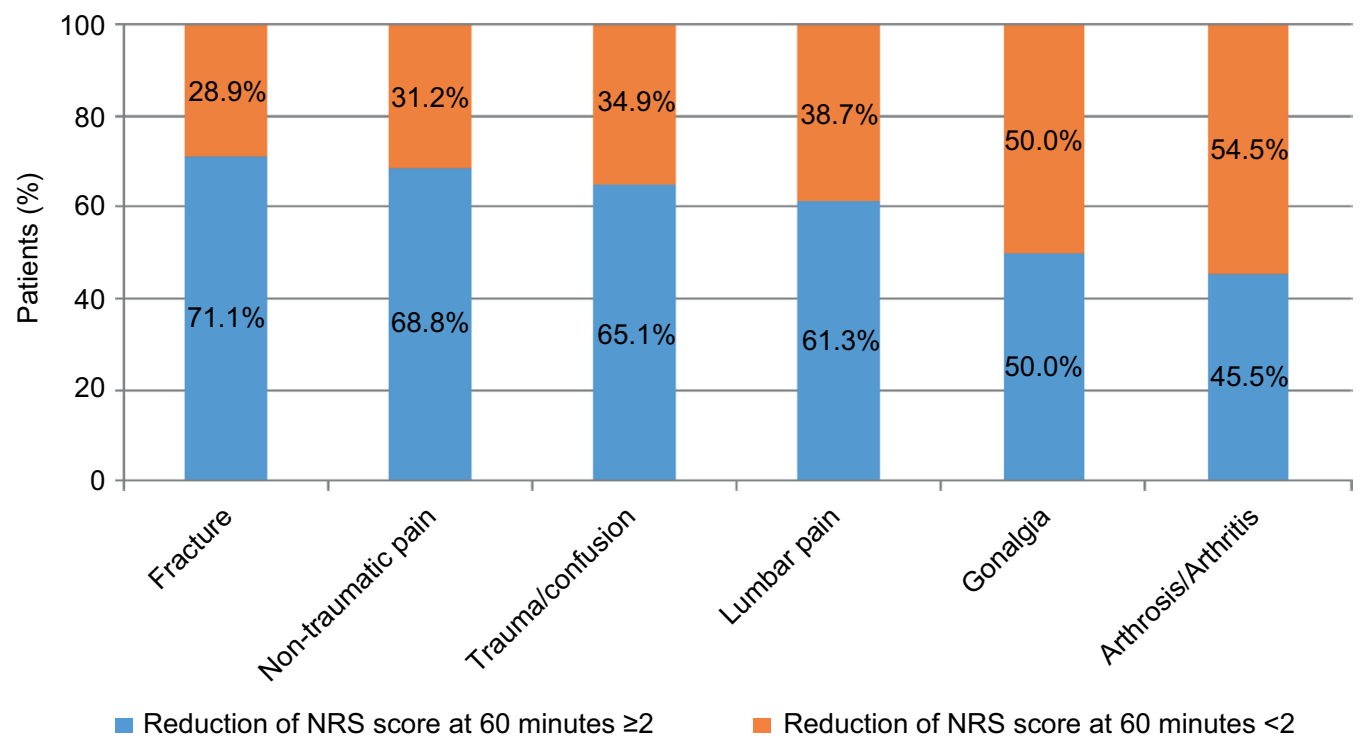

Figure 4 Diagnosis at discharge for pain reduction of NRS.

Abbreviation: NRS, Numeric Rating Scale.

Table 5 NRS score at enrolment vs NRS score at 60 minutes and at discharge ( $t$-test for paired samples)

\begin{tabular}{lllll}
\hline & Mean & N & dS & P-value \\
\hline NRS score at admission & 7.67 & 308 & 1.590 & $<0.000 \mathrm{I}$ \\
NRS score at 60 minutes & 5.44 & 308 & 2.247 & \\
NRS score at admission & 7.73 & 277 & 1.592 & $<0.000 \mathrm{I}$ \\
NRS score at discharge & 4.73 & 277 & 2.334 & \\
\hline
\end{tabular}

Abbreviation: NRS, Numeric Rating Scale.

where a propensity to the intake of analgesic therapy and protocol compliance ranging from $40 \%$ to $62 \%$ is highlighted. ${ }^{10-12}$

In total, there is a higher number of women (57.2\%) with a clearly greater mean age ( 52.8 vs 45.3 ). The predominant reason for admission is the pain resulting from trauma/contusion, which is also confirmed in the diagnosis at the time of discharge.

For what concerns the intensity of pain at admission, in our sample we can see a high number of cases with severe pain, 240 cases equal to $76.9 \%$, of which 72 (23.1\%) with NRS score equal to 10 , a score being higher than that found in similar studies. ${ }^{13}$ Despite the high number of patients with severe pain at admission, for what concerns the administered therapy, we can notice, however, that morphine was rarely used, being administered to only 8 patients $(2.6 \%)$. The fact that opioids are rarely used for pain management in the EDs is well known and well documented. ${ }^{10,14,15}$ In this context, among the developed countries, Italy ranks in the last place for opioid consumption. This gap from the rest of western countries can be imputed both to a lack of systematic training of health professionals regarding pain control, and to a sort of "opiophobia."6
Orosoluble paracetamol $1000 \mathrm{mg}$ represents the most widely used analgesic (97.4\%). Only $2.6 \%$ of the patients were treated with morphine vial $5 \mathrm{~mL} / 10 \mathrm{mg}$.

Globally, more than $65 \%$ of all detected clinical pictures show a reduction of at least 2 points on the NRS 1 hour after taking the analgesic. Averagely, the pain intensity is reduced by more than 2 points after 60 minutes, which become 3.29 at discharge, both comparisons to baseline being statistically significant $(P<0.0001)$.

The protocol is extremely efficient both for the time to analgesia and for the patient satisfaction. On average, the time from admission to the administration of analgesic drug is equal to $\sim 6$ minutes, with a median time of only 2 minutes, which falls to 1.5 in case of trauma and contusion. For only six patients, the time of intake of the analgesia is higher than 60 minutes.

The degree of patient satisfaction is high both at discharge and at the telephone interview carried out 2 days after the admission. For all items proposed at discharge, the degree of satisfaction is considerably higher than 9 points (maximum 10 points), where items proposed are the relationship between the patient and the triage nurse who verifies the presence of pain symptomatology, describes the protocol, proposes and administers the analgesic therapy. A total of 141 patients (45.6\%) expressed the highest level of satisfaction in reply to all questions submitted.

For what concerns the questionnaire asked at 48 hours after the discharge, which rather evaluates the organizational and logistic aspects, we still have a substantially positive evaluation $($ mean $=44.16$ maximum $=50)$, whereas some 
Table 6 Time of intake of the analgesic therapy by reason for admission (grouped)

\begin{tabular}{lllllll}
\hline Reason for admission & Time (minutes), mean & N & SD & Minimum & Maximum & Median \\
\hline Trauma/contusion & 5.5 & 168 & 20.749 & 0.00 & 189.00 & 1.5 \\
Lumbar pain + articular muscle & 5.8 & 91 & 17.744 & 0.00 & 142.00 & 2.0 \\
Other pain & 7.5 & 49 & 18.114 & 0.00 & 109.00 & 2.0 \\
Total & 5.9 & 308 & 19.449 & 0.00 & 189.00 & 2.0 \\
\hline
\end{tabular}

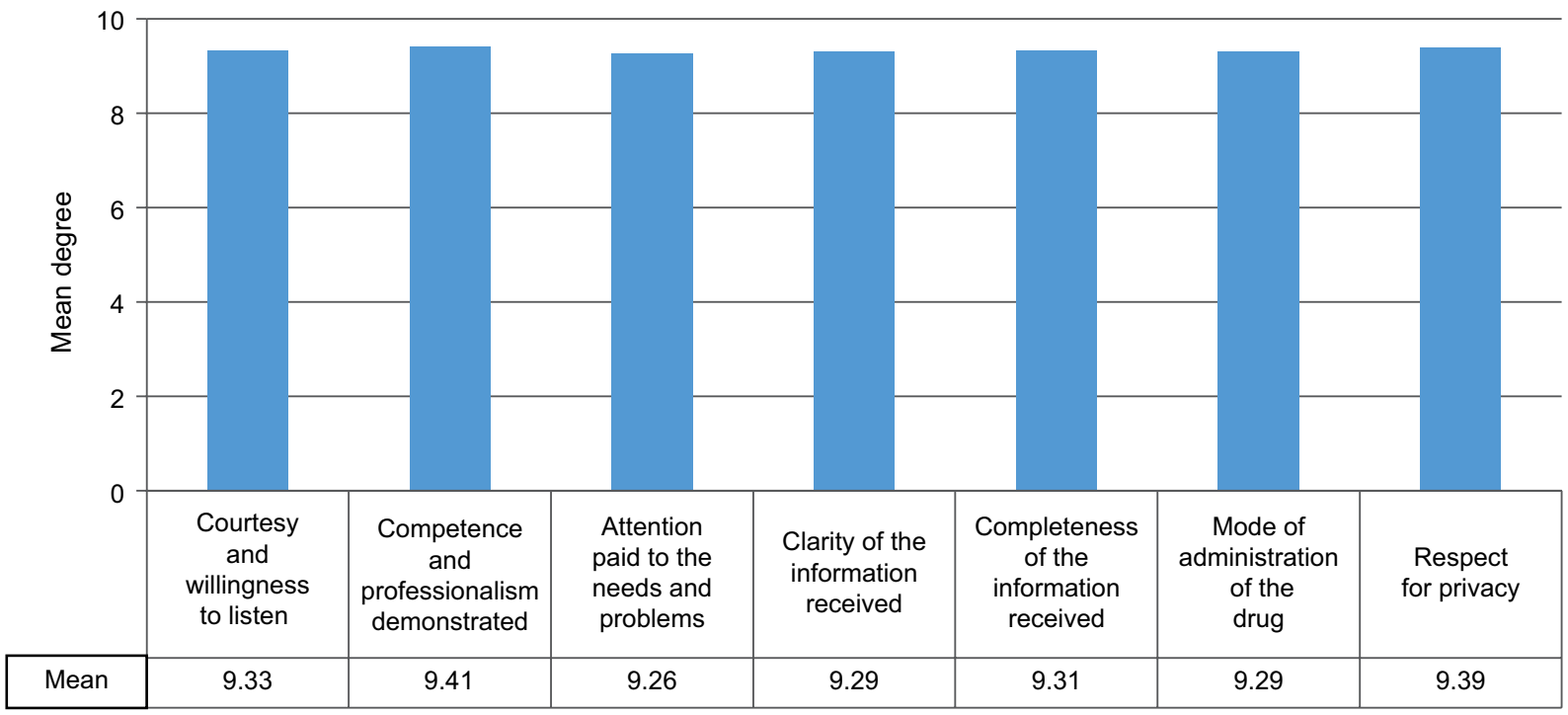

Figure 5 Degree of patient satisfaction detected at the time of discharge.

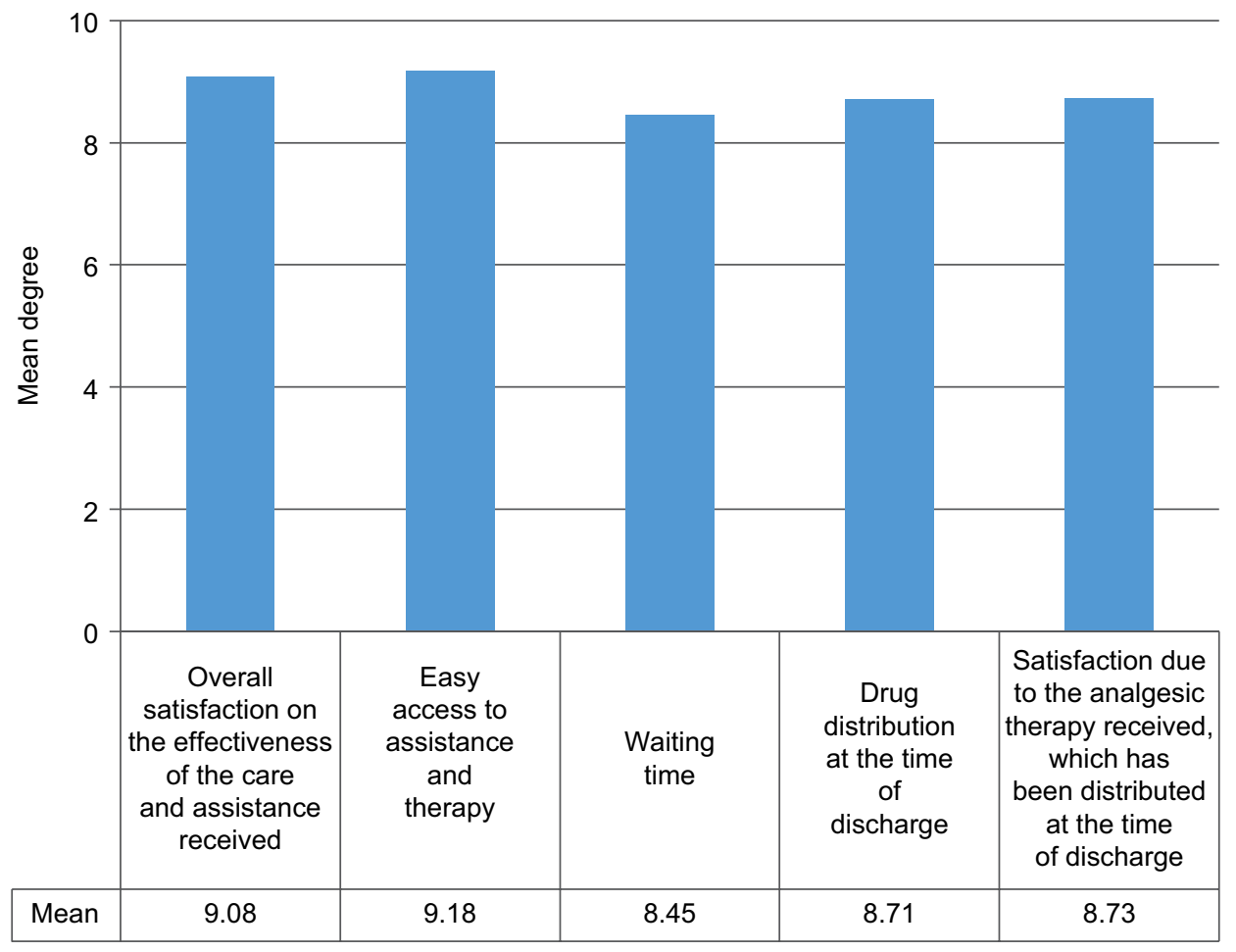

Figure 6 Degree of patient satisfaction detected at 48 hours after the discharge. 
critical aspects in relation to the waiting time and the therapy distribution mode at the time of discharge are evidenced.

\section{Conclusion}

This study shows the validity of the protocol adopted for the early treatment of the painful symptomatology in the ED in terms of both effectiveness and efficiency. The degree of pain control was significant and the degree of patient satisfaction was extremely elevated. The time of intake of the analgesia was extremely rapid, being mainly guaranteed by the easy handling of the orosoluble formulation of the paracetamol. This pharmaceutical form is particularly suitable for the emergency setting as it is ready to use and does not require the intake of water. The results of this study validate the effectiveness of orosoluble paracetamol $1000 \mathrm{mg}$ for the treatment of acute pain, especially pain due to trauma/contusion and lumbar/musculoarticular pain.

However, some critical points are detected, one being related to the pain assessment at admission, which highlights the presence of patients with severe pain markedly higher than that found in the literature, and the other being related to the rare use of opioids. Critical points could be overcome especially in terms of specific training of the operators. In the context of moderate-to-severe pain, the introduction of the opioid analgesics being equally effective as but more manageable than morphine, such as codeine in combination with paracetamol, in the pain management protocol in the triage, could ensure an improvement both in the protocol compliance and efficacy.

We hereby confirm the significant role of the doctor/nurse team in the implementation of pain management protocols during triage. The high degree of satisfaction, expressed also for the patient-nurse relationship in the triage area, confirms how the early pain management given by the triage nurse in the ED improves the quality of care offered to the patient.

\section{Acknowledgments}

We thank all the nurses of S.O.C. Pronto Soccorso Medicina d'Urgenza (Emergency Department), for their valuable contribution to the data collection. The results presented in this paper have not been published previously in whole or in part and are not under submission elsewhere. This submission represents an original work and all authors have read and approved the submission of this manuscript. The authors have no relevant conflicts of interest relating to the contents of this manuscript and have no relevant affiliations or financial involvement with any organization or entity with a financial interest in or financial conflict with the subject matter or materials discussed in the manuscript. The study received an unconditioned grant from A.C.R.A.F. Aziende Chimiche Riunite Angelini Francesco (S.P.A.) for the open access option.

\section{Author contributions}

All authors contributed in the study design and data interpretation, had accountable for all aspects of the work and approved the final manuscript as submitted. FP coordinated the study. LB, OB, RL, RB, SC, SDC, DDR and RLM wrote the initial draft. RLM performed the statistical analysis. All authors contributed toward data analysis, drafting and critically revising the paper and agree to be accountable for all aspects of the work.

\section{Disclosure}

The authors report no conflicts of interest in this work.

\section{References}

1. Berben SA, Meijs TH, van Dongen RT, et al. Pain prevalence and pain relief in trauma patients in the Accident \& Emergency department. Injury. 2008;39(5):578-585.

2. Rupp T, Delaney KA. Inadequate analgesia in emergency medicine. Ann Emerg Med. 2004;43(4):494-503.

3. Todd HK, Ducharme J, Choiniere M, et al; PEMI Study Group. Pain in the emergency department: results of the pain and emergency medicine initiative (PEMI) multicenter study. J Pain. 2007;8(6):460-466.

4. Fosnocht DE, Heaps ND, Swanson ER. Patient expectations for pain relief in the ED. Am J Emerg Med. 2004;22(4):286-288.

5. Wheeler E, Hardie T, Klemm P, et al. Level of pain and waiting time in the emergency department. Pain Manag Nurs. 2010;11(2):108-114.

6. Motov SM, Khan AN. Problems and barriers of pain management in the emergency department: are we ever going to get better? $J$ Pain Res. 2009;2:5-11.

7. Bergman CL. Emergency nurses' perceived barriers to demonstrating caring when managing adult patients' pain. J Emerg Nurs. 2012; 38(3):218-225.

8. Fosnocht DE, Swanson ER. Use of a triage pain protocol in the ED. Am J Emerg Med. 2007;25(7):791-793.

9. Savoia G, Coluzzi F, Di Maria C, et al. Italian intersociety recommendations on pain management in the emergency setting (SIAARTI, SIMEU, SIS 118, AISD, SIARED, SICUT, IRC). Minerva Anestesiol. 2015;81(2):205-225.

10. Allione A, Melchio R, Martini G, et al. Factors influencing desired and received analgesia in emergency department. Intern Emerg Med. 2011;6(1):69-78.

11. Stephan FP, Nickel CH, Martin JS, Grether D, Delport-Lehnen K, Bingisser R. Pain in the emergency department: adherence to an implemented treatment protocol. Swiss Med Wkly. 2010;140(23-24): 341-347.

12. Lecomte F, Huet S, Audureau E, Guyerdet V, Pourriat JL, Claessens YE. Patients in pain that refuse acetaminophen at triage. Am J Emerg Med. 2014;32(4):388-389.

13. Van Woerden, Van Den Brand CL, Den Hartog CF, Idenburg FJ, Grootendorst DC, Van Der Linden MC. Increased analgesia administration in emergency medicine after implementation of revised guidelines. Int J Emerg Med. 2016;9:4.

14. De Berardinis B, Laura Magrini L, Luigi Mario Castello LM, et al. Emergency department pain management and its impact on patients' short term outcome. Open Emerg Med J. 2013;5:1-7.

15. Gueant S, Taleb A, Borel-Kuhner J, et al. Quality of pain management in the emergency department: results of a multicenter prospective study. Eur J Anesthesiol. 2011;28(2):97-105. 


\section{Supplementary materials}

Table SI Satisfaction degree

\begin{tabular}{|c|c|c|c|c|c|c|c|c|c|c|c|c|}
\hline & & \multicolumn{11}{|c|}{ Min <-------------- Satisfaction -------------> Mas } \\
\hline \multicolumn{13}{|c|}{ Assessment of the degree of satisfaction at discharge } \\
\hline I & Courtesy and willingness to listen & 0 & I & 2 & 3 & 4 & 5 & 6 & 7 & 8 & 9 & 10 \\
\hline 2 & Competence and professionalism & 0 & I & 2 & 3 & 4 & 5 & 6 & 7 & 8 & 9 & 10 \\
\hline 3 & Attention to needs and problems & 0 & I & 2 & 3 & 4 & 5 & 6 & 7 & 8 & 9 & 10 \\
\hline 4 & Clarity of information received & 0 & 1 & 2 & 3 & 4 & 5 & 6 & 7 & 8 & 9 & 10 \\
\hline 5 & Thoroughness of information received & 0 & 1 & 2 & 3 & 4 & 5 & 6 & 7 & 8 & 9 & 10 \\
\hline 6 & Routes of administration of analgesic medication & 0 & I & 2 & 3 & 4 & 5 & 6 & 7 & 8 & 9 & 10 \\
\hline 7 & Compliance of privacy law & 0 & I & 2 & 3 & 4 & 5 & 6 & 7 & 8 & 9 & 10 \\
\hline \multicolumn{13}{|c|}{ Assessment of the degree of satisfaction after $48 \mathrm{~h}$ from the discharge } \\
\hline $\mathrm{I}$ & Overall satisfaction on the effectiveness of treatment and care received & 0 & I & 2 & 3 & 4 & 5 & 6 & 7 & 8 & 9 & 10 \\
\hline 2 & Ease of access to care and treatment & 0 & I & 2 & 3 & 4 & 5 & 6 & 7 & 8 & 9 & 10 \\
\hline 3 & Waiting list & 0 & I & 2 & 3 & 4 & 5 & 6 & 7 & 8 & 9 & 10 \\
\hline 4 & Drug distribution after discharge & 0 & I & 2 & 3 & 4 & 5 & 6 & 7 & 8 & 9 & 10 \\
\hline 5 & Overall satisfaction on analgesic therapy at the discharge & 0 & I & 2 & 3 & 4 & 5 & 6 & 7 & 8 & 9 & 10 \\
\hline
\end{tabular}

Table S2 Time after receipt of analgesic at discharge for each diagnosis

\begin{tabular}{lllllll}
\hline Diagnosis at discharge & Time (minutes), mean & N & SD & Min & Max & Mean \\
\hline Trauma contusion & 3.2 & 111 & 11,504 & 0.00 & 112.00 & 1.00 \\
Fracture & 8.9 & 39 & 31.288 & 0.00 & 189.00 & 2.00 \\
Nontraumatic pain & 8.6 & 95 & 23.108 & 0.00 & 142.00 & 2.00 \\
Lumbar pain & 2.9 & 31 & 5.531 & 0.00 & 30.00 & 2.00 \\
Arthrosis/arthritis & 3.8 & 12 & 2.918 & 1.00 & 10.00 & 2.50 \\
Gonalgia & 2.0 & 10 & 1.247 & 1.00 & 4.00 & 2.00 \\
Total & 5.6 & 298 & 18.830 & 1.00 & 189.00 & 2.00 \\
\hline
\end{tabular}

Journal of Pain Research

\section{Publish your work in this journal}

The Journal of Pain Research is an international, peer reviewed, open access, online journal that welcomes laboratory and clinical findings in the fields of pain research and the prevention and management of pain. Original research, reviews, symposium reports, hypothesis formation and commentaries are all considered for publication.
The manuscript management system is completely online and includes a very quick and fair peer-review system, which is all easy to use. Visit http://www.dovepress.com/testimonials.php to read real quotes from published authors. 